Chirurgia (2017) 112: 365-366

No. 4, July - August

Copyright $\odot$ Celsius

http://dx.doi.org/10.21614/chirurgia.112.4.365

\title{
How Can We Improve Education of Breast Surgeons Across Europe?
}

\section{Agnieszka Kolacinska}

Department of Surgical Oncology, Breast Unit, Department of Head and Neck Cancer Surgery, Medical University of Lodz Cancer Center, Lodz, Poland

Board of Directors of the European Society of Surgical Oncology (ESSO)

Examiner of the European Board of Surgery Qualification in Breast Surgery

Corresponding author: Agnieszka Kolacinska, MD Department of Surgical Oncology Breast Unit, Department of Head and Neck Cancer Surgery Medical University of Lodz Cancer Center, Lodz, Poland E-mail: agnieszka.kolacinska@umed.lodz.pl
Received: 27.03 .2017 Accepted: 30.04 .2017
- The proposed global curriculum developed by the American Society of Surgical Oncology (SSO) and the European Society of Surgical Oncology (ESSO) and the textbook: provides a state-of- the- art of breast cancer surgery, complements the syllabus and curriculum of the Union Européenne des Médecins Spécialistes (UEMS) examination in breast surgery/ European Board of Surgery Qualification in Breast Surgery (EBSQinBS) administered by ESSO (1)

- Knowledge and understanding of the principles of breast cancer incidence, aetiology, risk factors, genetics, premalignant and high-risk lesions, pathology, prognostic and predictive factors, risk prediction models, screening, diagnosis and imaging, breast cancer, oncoplastic, reconstructive breast surgery, axillary surgery and future perspective for this, radiation therapy, neoadjuvant and adjuvant systemic treatment- endocrine-, immuno- and chemotherapy, side effects and toxicities of treatment, locoregional recurrence, follow-up, locally advanced breast cancer, metastatic breast cancer, breast cancer in young and elderly patients, survivor issues, lymphedema, palliative care, chronić pain, body image and sexuality, fertility issues, cognitive functions, etc.) (1)

- ESSO fellowships: for instance BSSO (Brazilian Society of Surgical Oncology)-ESSO fellowship, ESSO members are being offered the opportunity by the BSSO to apply for a visiting observership in Brazil; ESSO fellowship in Breast Surgery- the fellowship provides further specialised training in 
the multi-modality clinical care specific to the breast cancer patient and a deeper training in breast cancer research; training fellowships- to allow young surgeons to visit a specialist breast unit in Europe, to help them to expand their experience and learn new techniques; congress fellowships- for each ESSO congress the ESSO Scientific Committee awards a number of fellowship grantsto participants from low-income countries to attend the congress; EYSAC (ESSO Young Surgeons and Alumni Club) research fellowship; SSO/ ESSO fellowshipthrough its partnership with ESSO, SSO provides a grant to attend the SSO Annual Symposium; Reciprocal International Exchange Programmes with Japan, Latin America and North America (2).

- ESSO courses and masterclasses: expanded portfolio of courses, in- and outside Europe, basic and advanced, international faculty, multidisciplinary approach, learning methods: hands-on with human cadavers, workshops in small groups, live and case demonstrations, interactive sessions, 3D videos (for instance in 2017 ESSO course on ultrasound of breast in Barcelona, advanced course on oncoplastic surgery in Barcelona, advanced course on breast cancer surgery in Naples) (2).

- Trials: Interantional Nipple- Sparing Mastectomy Registry (INSPIRE), EURECCA (European Cancer Audit) project. The aim of INSPIRE is to provide pooled evidence derived from a prospective collaborative high-quality registry between international centers, oncological safety, patient- reported outcome measures, launched in March, 2016, European Breast Cancer Conference EBCC 10, Amsterdam (2).

- Examination: European Board of Surgery Qualification in Breast Surgery (EBSQ in $\mathrm{BS})$ : phase 1-eligibility assessment (CV, logbook, references); phase 2- test; phase 3- oral examination with 2 clinical cases and 1 critical review of academic paper. The applicant must hold a current license to practice as a surgeon (general or plastic or gynaecologist), demonstrate work for one year in a breast surgery unit with at least 150 new primary breast cancer cases per year, attended at least one national/ international training course in breast surgery and has attended at least one well recognized international congress. The applicant should present a signed log book for breast surgery with application; published either one paper or book chapter on breast disease (2).

\section{Conclusions}

1. The need for certified breast units: Standardised techniques by specialist breast surgeons across Europe should be the aim.

2. No man's land. Across Europe oncoplastic reconstruction is performed by specialist breast, general or plastic surgeons, sometimes in collaboration with each other. There may be dispute as who should or should not be involved. This needs to be addressed.

3. Oncoplastic reconstruction technique expansion. Significant expansion in number, variety and complexity of available techniques is a fact. A good example would be a 6 -fold increase together with a low rate of complications in some Eastern European countries over the last decade

4. CME. European breast surgeons want to improve their skills, as evidenced by fully or overbooked ESSO-endorsed breast surgery courses and other high quality workshops. Applications for the European Examination in Breast Surgery (EBSQBS) continue to increase.

\section{Refences}

1. Are C, Berman RS, Wyld L, Cummings C, Lecoq C, Audisio RA. Global curriculum in surgical oncology. Eur J Surg Oncol. 2016; 42(6):754-66. doi: 10.1016/j.ejso.2016.04.005. Epub 2016 May 1.

2. www.essoweb.org 